\title{
Some New Results of Mitrinović-Cusa's and Related Inequalities Based on the Interpolation and Approximation Method
}

\author{
Lina Zhang ${ }^{1}$ and Xuesi Ma $\mathbb{D}^{2}$ \\ ${ }^{1}$ School of Computer Science and Technology, Henan Polytechnic University, Jiaozuo, Henan 454000, China \\ ${ }^{2}$ School of Mathematics and Information Science, Henan Polytechnic University, Jiaozuo, Henan 454000, China \\ Correspondence should be addressed to Xuesi Ma; maxuesi@hpu.edu.cn
}

Received 25 February 2021; Revised 22 March 2021; Accepted 25 March 2021; Published 15 April 2021

Academic Editor: Dimitri Mugnai

Copyright (c) 2021 Lina Zhang and Xuesi Ma. This is an open access article distributed under the Creative Commons Attribution License, which permits unrestricted use, distribution, and reproduction in any medium, provided the original work is properly cited.

In this paper, new refinements and improvements of Mitrinović-Cusa's and related inequalities are presented. First, we give new polynomial bounds for $\operatorname{sinc}(x)$ and $\cos (x)$ functions using the interpolation and approximation method. Based on the obtained results of the above two functions, we establish new bounds for Mitrinović-Cusa's, Wilker's, Huygens', Wu-Srivastava's, and Neuman-Sándor's inequalities. The analysis results show that our bounds are tighter than the previous methods.

\section{Introduction}

Trigonometric inequalities play an important role in pure and applied mathematics and have been used in various fields. This study starts from the following inequality:

$$
(\cos (x))^{(1 / 3)}<\sin c(x)=\frac{\sin (x)}{x}<\frac{2+\cos (x)}{3}, \quad 0<x<\frac{\pi}{2}
$$

which is the focus of many researchers. The left-side inequality of (1) was first proved by Mitrinović [1,2], which is called as Mitrinović-s inequality. The right-side inequality of (1) was proposed by the German philosopher and theologian Nicolaus de Cusa and proved explicitly by Huygens [3]. Therefore, inequality (1) is called as Mitrinović-Cusa's inequality.

Huygens' inequality is

$$
2 \frac{\sin (x)}{x}+\frac{\tan (x)}{x}>3, \quad 0<x<\frac{\pi}{2}
$$

which is a consequence of inequality (1) [3].

The following inequality

$$
\left(\frac{\sin (x)}{x}\right)^{2}+\frac{\tan (x)}{x}>2, \quad 0<x<\frac{\pi}{2},
$$

due to Wilker, is called as Wilker's inequality [4].

$\mathrm{Wu}$ and Srivastava [5] proved the following inequality:

$$
\left(\frac{x}{\sin (x)}\right)^{2}+\frac{x}{\tan (x)}>2, \quad 0<x<\frac{\pi}{2} \text {. }
$$

Neuman and Sándor [6] presented the following inequality:

$$
3 \frac{x}{\sin (x)}+\cos (x)>4, \quad 0<x<\frac{\pi}{2} .
$$

Inequalities (1)-(5) have attracted the attention of many scholars [7-32].

Bhayo and Sándor [33] obtained the following results, for $x \in(0,(\pi / 2))$ :

$$
\begin{gathered}
\frac{2+\cos (x)-(x / \pi)^{3}}{3}<\frac{\sin (x)}{x}<\frac{2+\cos (x)-(x / \pi)^{4}}{3}, \\
\frac{\sin (x)}{x}<\frac{\pi^{2}-x^{2}}{\pi^{2}+\alpha x^{2}}<\frac{2+\cos (x)}{3}<\frac{\pi^{2}-x^{2}}{\pi^{2}+\beta x^{2}}
\end{gathered}
$$


where $\alpha=\left(\pi^{2} / 6\right)-1$ and $\beta=(1 / 2)$ are the best possible constants.

Yang [34] gave the following inequalities:

$$
\begin{aligned}
& \frac{\sin (x)}{x}>(\cos (p x))^{1 /\left(3 p^{2}\right)}, \quad 0<x<\frac{\pi}{2}, p \in\left[p_{1}, 1\right], \\
& \frac{\sin (x)}{x}<(\cos (q x))^{1 /\left(3 q^{2}\right)}, \quad 0<x<\frac{\pi}{2}, q \in(0,(\sqrt{5} / 5)],
\end{aligned}
$$

where $p_{1}=0.45346830977067 \ldots$ is the unique root of equation $\ln (2 / \pi)-\left(1 / 3 p^{2}\right) \ln \cos (p \pi / 2)=0$ in $p \in(0,1]$.

Yang [35] gave further results:

$$
\begin{aligned}
\frac{2 p+(p+3) \cos (x)}{(3 p+1)+2 \cos (x)}< & (\cos (x))^{(1 / 3)}<\frac{2 q+(q+3) \cos (x)}{(3 q+1)+2 \cos (x)} \\
& <\frac{\sin (x)}{x} \\
& <\frac{2 r+(r+3) \cos (x)}{(3 r+1)+2 \cos (x)}<\frac{2+\cos (x)}{3} \\
& <\frac{2 s+(s+3) \cos (x)}{(3 s+1)+2 \cos (x)}
\end{aligned}
$$

where $x \in(0,(\pi / 2)), p=0, q \in\left[1,(\pi-3)^{-1}\right], r \in[9, \infty]$, and $s \in[-\infty,-1]$.

Sumner et al. [36] obtained a further result of Wilker's inequality as follows:

$$
\begin{array}{r}
2+\frac{16}{\pi^{4}} x^{3} \tan (x)<\left(\frac{\sin (x)}{x}\right)^{2}+\frac{\tan (x)}{x}<2+\frac{8}{45} x^{3} \tan (x) \\
0<x<\frac{\pi}{2}
\end{array}
$$

Furthermore, $\left(16 / \pi^{4}\right)$ and $(8 / 45)$ are the best constants. Chen and Cheung [37] proved the following inequalities, for $x \in(0,(\pi / 2))$ :

$$
\begin{gathered}
3+\frac{3}{20} x^{3} \tan (x)<2 \frac{\sin (x)}{x}+\frac{\tan (x)}{x}<3+\left(\frac{2}{\pi}\right)^{4} x^{3} \tan (x), \\
3+\frac{3}{20} x^{4}+\frac{3}{56} x^{5} \tan (x)<2 \frac{\sin (x)}{x}+\frac{\tan (x)}{x}<3+\frac{3}{20} x^{4}+\left(\frac{2}{\pi}\right)^{6} x^{5} \tan (x), \\
2+\frac{8}{45} x^{4}+\frac{16}{315} x^{5} \tan (x)<\left(\frac{\sin (x)}{x}\right)^{2}+\frac{\tan (x)}{x}<2+\frac{8}{45} x^{4}+\left(\frac{2}{\pi}\right)^{6} x^{5} \tan (x), \\
2+\frac{8}{45} x^{4}+\frac{16}{315} x^{6}+\frac{104}{4725} x^{7} \tan (x)<\left(\frac{\sin (x)}{x}\right)^{2}+\frac{\tan (x)}{x}<2+\frac{8}{45} x^{4}+\frac{16}{315} x^{6}+\left(\frac{2}{\pi}\right)^{8} x^{7} \tan (x), \\
\left(\frac{x}{\sin (x)}\right)^{2}+\frac{x}{\tan (x)}<2+\frac{2}{45} x^{3} \tan (x), \\
\left(\frac{x}{\sin (x)}\right)^{2}+\frac{x}{\tan (x)}<2+\frac{2}{45} x^{4}+\frac{8}{945} x^{5} \tan (x),
\end{gathered}
$$

where $(3 / 20),(2 / \pi)^{4},(3 / 56),(2 / \pi)^{6},(16 / 315),(104 / 4725)$, $(2 / \pi)^{8},(2 / 45)$, and $(8 / 945)$ are the best possible constants.
Nenezic et al. [38] proved the following inequalities, for $x \in(0,(\pi / 2))$ : 


$$
\begin{aligned}
2+\left(\frac{8}{45}-\frac{8}{945} x^{2}\right) x^{3} \tan (x) & <\left(\frac{\sin (x)}{x}\right)^{2}+\frac{\tan (x)}{x} \\
& <2+\left(\frac{8}{45}-\frac{8}{945} x^{2}+\frac{480 \pi^{6}-40320 \pi^{4}+3628800}{14175 \pi^{8}} x^{4}\right) x^{3} \tan (x), \\
2+\left(\frac{16}{\pi^{4}}+\left(\frac{160}{\pi^{5}}-\frac{16}{\pi^{3}}\right)\left(\frac{\pi}{2}-x\right)\right) x^{3} \tan (x) & <\left(\frac{\sin (x)}{x}\right)^{2}+\frac{\tan (x)}{x} \\
& <2+\left(\frac{16}{\pi^{4}}+\left(\frac{160}{\pi^{5}}-\frac{16}{\pi^{3}}\right)\left(\frac{\pi}{2}-x\right)+\left(\frac{960}{\pi^{6}}-\frac{96}{\pi^{4}}\right)\left(\frac{\pi}{2}-x\right)^{2}\right) x^{3} \tan (x) .
\end{aligned}
$$

Chen and Paris [39] gave the following inequalities, for $x \in(0,(\pi / 2))$ :

$$
\begin{aligned}
& 3+\left(\frac{3}{20}+\frac{1}{280} x^{2}+\frac{23}{33600} x^{4}\right) x^{3} \tan (x)<2 \frac{\sin (x)}{x}+\frac{\tan (x)}{x}<3+\left(\frac{3}{20}+\frac{1}{280} x^{2}+\frac{17920-168 \pi^{4}-\pi^{6}}{70 \pi^{8}} x^{4}\right) x^{3} \tan (x) \\
& 2+\left(\frac{8}{45}-\frac{8}{945} x^{2}+\frac{16}{14175} x^{4}\right) x^{3} \tan (x)<\left(\frac{\sin (x)}{x}\right)^{2}+\frac{\tan (x)}{x}<2+\left(\frac{8}{45}-\frac{8}{945} x^{2}+\frac{241920-2688 \pi^{4}+32 \pi^{6}}{945 \pi^{8}} x^{4} x^{3} \tan (x)\right. \\
& 2+\left(\frac{2}{45}-\frac{2}{315} x^{2}-\frac{224-8 \pi^{2}}{315 \pi^{4}} x^{4}\right) x^{3} \tan (x)<\left(\frac{x}{\sin (x)}\right)^{2}+\frac{x}{\tan (x)}<2+\left(\frac{2}{45}-\frac{2}{315} x^{2}-\frac{4}{1575} x^{4}\right) x^{3} \tan (x) \\
& \text { Wang [40] proved the following inequality: } \quad 4+\frac{1}{10} x^{3} \sin (x)<3 \frac{x}{\sin (x)}+\cos (x)<4+\frac{12 \pi-32}{\pi^{3}} x^{3} \sin (x)
\end{aligned}
$$

$$
\begin{aligned}
2+\frac{2}{45} x^{3} \sin (x)< & \left(\frac{x}{\sin (x)}\right)^{2}+\frac{x}{\tan (x)}<2 \\
& +\left(\frac{2}{\pi}-\frac{16}{\pi^{3}}\right) x^{3} \sin (x), \quad 0<x<\frac{\pi}{2},
\end{aligned}
$$

where $(2 / 45)$ and $(2 / \pi)-\left(16 / \pi^{3}\right)$ are the best possible constants.

Jiang et al. [41] gave the following inequality:

$$
\begin{aligned}
&-\frac{x^{4}}{15}<\cos (x)-\left(\frac{\sin (x)}{x}\right)^{3}<-\frac{x^{4}}{15}+\frac{23 x^{6}}{1890}, \\
&-\frac{1}{180} x^{4}<\frac{\sin (x)}{x}-\frac{\cos (x)+2}{3}<-\frac{1}{180} x^{4}+\frac{1}{3780} x^{6}, \\
& 3+\left(\frac{3}{20} x^{4}-\frac{3}{140} x^{6}\right) \frac{1}{\cos (x)}<2 \frac{\sin (x)}{x}+\frac{\tan (x)}{x}<3+\frac{3}{20} \frac{x^{4}}{\cos (x)}, \\
& 2+\left(\frac{8}{45} x^{4}-\frac{8}{105} x^{6}\right) \frac{1}{\cos (x)}<\left(\frac{\sin (x)}{x}\right)^{2}+\frac{\tan (x)}{x}<2+\frac{8}{45} \frac{x^{4}}{\cos (x)},
\end{aligned}
$$




$$
\begin{aligned}
& \left(\frac{x}{\sin (x)}\right)^{2}+\frac{x}{\tan (x)}>2+\frac{2}{45} x^{4}, \\
& 3 \frac{x}{\sin (x)}+\cos (x)>4+\frac{1}{10} x^{4}+\frac{1}{210} x^{6} .
\end{aligned}
$$

Maleševic et al. [43] further improved the above results of inequalities (24)-(29), for $x \in(0,(\pi / 2))$ and $n \in N$ :

$$
\begin{aligned}
& \sum_{k=2}^{2 n}(-1)^{k} A(x) x^{2 k}<\cos (x)-\left(\frac{\sin (x)}{x}\right)^{3}<\sum_{k=2}^{2 n+1}(-1)^{k} A(k) x^{2 k} \\
& \sum_{k=2}^{2 n}(-1)^{k+1} B(x) x^{2 k}<\frac{\sin (x)}{x}-\frac{\cos (x)+2}{3}<\sum_{k=2}^{2 n+1}(-1)^{k+1} B(k) x^{2 k} \\
& 3+\frac{1}{\cos (x)} \sum_{k=2}^{2 n+1}(-1)^{k} C(k) x^{2 k}<2 \frac{\sin (x)}{x}+\frac{\tan (x)}{x}<3+\frac{1}{\cos (x)} \sum_{k=2}^{2 n}(-1)^{k} C(k) x^{2 k}, \\
& 2+\frac{1}{\cos (x)} \sum_{k=2}^{2 n+1}(-1)^{k} D(x) x^{2 k}<\left(\frac{\sin (x)}{x}\right)^{2}+\frac{\tan (x)}{x}<2+\frac{1}{\cos (x)} \sum_{k=2}^{2 n}(-1)^{k} D(k) x^{2 k}, \\
& 2+\sum_{k=2}^{m-1} \frac{\left|\mathbf{B}_{2 k}\right|(2 k-2) 4^{k}}{(2 k) !} x^{2 k}+\left(\frac{2 x}{\pi}\right)^{2 m}\left(\frac{\pi^{2}}{4}-2-\sum_{k=2}^{m-1} \frac{\left|\mathbf{B}_{2 k}\right|(2 k-2) 4^{k}}{(2 k) !}\left(\frac{\pi}{2}\right)^{2 k}\right)>\left(\frac{x}{\sin (x)}\right)^{2}+\frac{x}{\tan (x)}>2+\sum_{k=2}^{m} \frac{\left|\mathbf{B}_{2 k}\right|(2 k-2) 4^{k}}{(2 k) !} x^{2 k} \\
& 4+\sum_{k=1}^{m-1} \frac{3\left|\mathbf{B}_{2 k}\right|\left(2^{2 k}-2\right)+(-1)^{k}}{(2 k) !} x^{2 k}+\left(\frac{2 x}{\pi}\right)^{2 m}\left(\frac{3 \pi}{2}-4-\sum_{k=1}^{m-1} \frac{3\left|\mathbf{B}_{2 k}\right|\left(2^{2 k}-2\right)+(-1)^{k}}{(2 k) !}\left(\frac{\pi}{2}\right)^{2 k}\right) \\
& >3 \frac{x}{\sin (x)}+\cos (x)>4+\sum_{k=1}^{m} \frac{3\left|\mathbf{B}_{2 k}\right|\left(2^{2 k}-2\right)+(-1)^{k}}{(2 k) !} x^{2 k},
\end{aligned}
$$

where $A(k)=\left(3^{2 k+3}-32 k^{3}-96 k^{2}-88 k-27 / 4(2 k+3) !\right)$, $B(k)=(2 / 3)(k-1 /(2 k+1) !), C(k)=2\left(4^{k}-3 k-1 / \quad(2 k+\right.$ $1) !), D(k)=(1 / 4)\left(-9+3^{2 k+2}-40 k-32 k^{2} /(2 k+2) !\right)$, and $\mathbf{B}_{i}$ are Bernoulli's numbers.

The goal of this paper is to obtain some new inequalities which provide generalizations of inequalities (1)-(5). In order to provide the new bounds of Mitrinović-Cusa's and related inequalities, we introduce a method called the interpolation and approximation. This method has been successfully applied to prove and approximate a wide category of trigonometric inequalities $26,27,44-46$.

In this paper, we first give the new polynomial bounds of $\sin c(x)$ and $\cos (x)$ functions using the interpolation and approximation method. We obtain the new bounds of Mitrinović-Cusa's and related inequalities based on the new bounds of the above two functions. The related inequalities include, Wilker's, Huygens', Wu-Srivastava's, and Neuman-Sandor's inequalities. At the same time, we also directly use the interpolation and approximation method to get the upper and lower bounds of Mitrinović-Cusa's inequality. The analysis results show that our bounds are tighter than the previous conclusions.

\section{Main Results}

Firstly, we introduce the following theorem of interpolation and approximation which is very useful for our proof [47].

Theorem 1. Let $w_{0}, w_{1}, \ldots, w_{r}$ be $r+1$ distinct points in $[a, b]$ and $n_{0}, n_{1}, \cdots n_{r}$ be $r+1$ integers $\geq 0$. Let $N=n_{0}+\cdots+n_{r}+r$. Suppose that $g(t)$ is a polynomial of degree $N$ such that

$$
g^{(i)}\left(w_{j}\right)=f^{(i)}\left(w_{j}\right), \quad i=0, \ldots, n_{j}, j=0, \ldots, r .
$$

Then, there exists $\xi(t) \in[a, b]$ such that 


$$
f(t)-g(t)=\frac{f^{(N+1)}(\xi(t))}{(N+1) !} \prod_{i=0}^{r}\left(t-w_{i}\right)^{n_{i}+1} .
$$

Next, we try to derive the novel polynomial bounds of $\sin c(x)$ and $\cos (x)$ functions using the above interpolation and approximation theorem.

Theorem 2. For $x \in(0, \pi / 2)$, we have that

$$
\begin{aligned}
s_{l}(x) & =1+b_{2} x^{2}+b_{3} x^{3}+b_{4} x^{4}+b_{5} x^{5}+b_{6} x^{6}+b_{7} x^{7}+b_{8} x^{8} \leq \sin c(x) \\
& \leq 1+a_{1} x+a_{2} x^{2}+a_{3} x^{3}+a_{4} x^{4}+a_{5} x^{5}+a_{6} x^{6}+a_{7} x^{7}+a_{8} x^{8}=s_{u}(x),
\end{aligned}
$$

where

$$
\begin{aligned}
& a_{1}=\frac{1}{\pi^{2}}\left[-5768+2048 \sqrt{2}+8019 \frac{\sqrt{3}}{2}-\left(\frac{303}{2} 2+512 \sqrt{2}+243 \sqrt{3}\right) \pi\right], \\
& a_{2}=\frac{1}{\pi^{3}}[146932-45056 \sqrt{2}-107163 \sqrt{3}+(7331 / 2+13312 \sqrt{2}+5832 \sqrt{3}) \pi], \\
& a_{3}=\frac{1}{\pi^{4}}[-1542128+395264 \sqrt{2}+1192644 \sqrt{3}-(39696+143872 \sqrt{2}+58563 \sqrt{3}) \pi], \\
& a_{4}=\frac{1}{\pi^{5}}[8694420-1769472 \sqrt{2}-7155864 \sqrt{3}+(236620+837632 \sqrt{2}+319302 \sqrt{3}) \pi], \\
& a_{5}=\frac{1}{\pi^{6}}[-28539504+4276224 \sqrt{2}+24978456 \sqrt{3}-(828216+2838528 \sqrt{2}+1021572 \sqrt{3}) \pi], \\
& a_{6}=\frac{1}{\pi^{7}}[54670032-5308416 \sqrt{2}-50703408 \sqrt{3}+(1694232+5603328 \sqrt{2}+1918728 \sqrt{3}) \pi], \\
& a_{7}=\frac{1}{\pi^{8}}[-56681856+2654208 \sqrt{2}+55427328 \sqrt{3}-(1871424+5971968 \sqrt{2}+1959552 \sqrt{3}) \pi], \\
& a_{8}=\frac{1}{\pi^{9}}[24572160-25194240 \sqrt{3}+(860544+2654208 \sqrt{2}+839808 \sqrt{3}) \pi], \\
& b_{2}=\frac{1}{\pi^{3}}\left[-14572+12288 \sqrt{2}+5103 \sqrt{3}-\left(\frac{1153}{2}+1024 \sqrt{2}+972 \sqrt{3}\right) \pi\right], \\
& b_{3}=\frac{1}{\pi^{4}}\left[355544-278528 \sqrt{2}-252963 \sqrt{3} / 2+\left(\frac{20295}{2}+24576 \sqrt{2}+21384 \sqrt{3}\right) \pi\right] \text {, } \\
& b_{4}=\frac{1}{\pi^{5}}[-3429916+2535424 \sqrt{2}+1272105 \sqrt{3}-(81833+238592 \sqrt{2}+191484 \sqrt{3}) \pi], \\
& b_{5}=\frac{1}{\pi^{6}}[16935408-11870208 \sqrt{2}-6632442 \sqrt{3}+(366210+1198080 \sqrt{2}+894240 \sqrt{3}) \pi], \\
& b_{6}=\frac{1}{\pi^{7}}[-45416304+30228480 \sqrt{2}+18869436 \sqrt{3}-(934596+3280896 \sqrt{2}+2297808 \sqrt{3}) \pi], \\
& b_{7}=\frac{1}{\pi^{8}}[62923392-39813120 \sqrt{2}-27713664 \sqrt{3}+(1270080+4644864 \sqrt{2}+3079296 \sqrt{3}) \pi] \text {, } \\
& b_{8}=\frac{1}{\pi^{9}}[-35230464+21233664 \sqrt{2}+16376256 \sqrt{3}-(710208+2654208 \sqrt{2}+1679616 \sqrt{3}) \pi] .
\end{aligned}
$$


Proof. Let $e_{s l}(x)=\sin c(x)-1-b_{2} x^{2}-b_{3} x^{3}-b_{4} x^{4}-$ $b_{5} x^{5}-b_{6} x^{6}-b_{7} x^{7}-b_{8} x^{8} \quad$ and $\quad e_{s u}(x)=\sin c(x)-1-$ $a_{1} x-a_{2} x^{2}-a_{3} x^{3}-a_{4} x^{4}-a_{5} x^{5}-a_{6} x^{6}-a_{7} x^{7}-a_{8} x^{8}$; then, $e_{s l}^{(9)}(x)=e_{s u}^{(9)}(x)=\sin c^{(9)}(x)$.

It is easy to see that

$\sin c^{(9)}(x)=\frac{\left(-9 x^{8}+504 x^{6}-15120 x^{4}+181440 x^{2}-362880\right) \sin (x)+\left(x^{9}-72 x^{7}+3024 x^{5}-60480 x^{3}+362880 x\right) \cos (x)}{x^{10}}$.

We let $g(x)=\left(-9 x^{8}+504 x^{6}-15120 x^{4}+181440 x^{2}-\right.$ $362880) \sin (x)+\left(x^{9}-72 x^{7}+3024 x^{5}-60480 x^{3}+362880 x\right)$ $\cos (x)$; then,

$$
g^{\prime}(x)=-x^{9} \sin (x)<0, \quad 0<x<\frac{\pi}{2} .
$$

Therefore, $g(x)$ is a monotone decreasing function in $(0,(\pi / 2))$, and we have $g(x) \leq g(0)=0$; then, $\sin c^{(9)}(x) \leq$ 0 for $x \in(0,(\pi / 2))$.

By the definition of $e_{s l}(x)$ and $e_{s u}(x)$, we have

$$
\begin{aligned}
& e_{s u}(0)=e_{s u}\left(\frac{\pi}{6}\right)=e_{s u}\left(\frac{\pi}{4}\right)=e_{s u}\left(\frac{\pi}{3}\right)=e_{s u}\left(\frac{\pi}{2}\right)=e_{s u}^{\prime}\left(\frac{\pi}{6}\right)=e_{s u}^{\prime}\left(\frac{\pi}{4}\right)=e_{s u}^{\prime}\left(\frac{\pi}{3}\right)=e_{s u}^{\prime}\left(\frac{\pi}{2}\right)=0 \\
& e_{s l}(0)=e_{s l}\left(\frac{\pi}{6}\right)=e_{s l}\left(\frac{\pi}{4}\right)=e_{s l}\left(\frac{\pi}{3}\right)=e_{s l}\left(\frac{\pi}{2}\right)=e_{s l}^{\prime}(0)=e_{s l}^{\prime}\left(\frac{\pi}{6}\right)=e_{s l}^{\prime}\left(\frac{\pi}{4}\right)=e_{s l}^{\prime}\left(\frac{\pi}{3}\right)=0 .
\end{aligned}
$$

By Theorem 1, there exist $\zeta_{j}, j=1,2$, such that

$$
\begin{gathered}
e_{s u}(x)=\frac{\sin c^{(9)}\left(\zeta_{1}\right)}{9 !} x\left(x-\frac{\pi}{6}\right)^{2}\left(x-\frac{\pi}{4}\right)^{2}\left(x-\frac{\pi}{3}\right)^{2}\left(x-\frac{\pi}{2}\right)^{2} \leq 0, \\
e_{s l}(x)=\frac{\sin c^{(9)}\left(\zeta_{2}\right)}{9 !} x^{2}\left(x-\frac{\pi}{6}\right)^{2}\left(x-\frac{\pi}{4}\right)^{2}\left(x-\frac{\pi}{3}\right)^{2}\left(x-\frac{\pi}{2}\right) \geq 0,
\end{gathered}
$$

which mean the conclusions are valid.

The proof of Theorem 2 is completed.

$$
\begin{aligned}
c_{l}(x) & =1+d_{2} x^{2}+d_{3} x^{3}+d_{4} x^{4}+d_{5} x^{5}+d_{6} x^{6}+d_{7} x^{7}+d_{8} x^{8} \leq \cos (x) \\
& \leq 1+c_{1} x+c_{2} x^{2}+c_{3} x^{3}+c_{4} x^{4}+c_{5} x^{5}+c_{6} x^{6}+c_{7} x^{7}+c_{8} x^{8}=c_{u}(x),
\end{aligned}
$$

where

$$
\begin{aligned}
& c_{1}=\frac{1}{\pi}\left[1185-1215 \sqrt{3}+\left(\frac{83}{2}+128 \sqrt{2}+81 \frac{\sqrt{3}}{2}\right) \pi\right], \\
& c_{2}=\frac{1}{\pi^{2}}\left[-\frac{64111}{2}+2048 \sqrt{2}+30618 \sqrt{3}-\left(1000+3328 \sqrt{2}+2187 \frac{\sqrt{3}}{2}\right) \pi\right], \\
& c_{3}=\frac{1}{\pi^{3}}\left[357852-45056 \sqrt{2}-319059 \sqrt{3}+\left(\frac{20179}{2}+35968 \sqrt{2}+12312 \sqrt{3}\right) \pi\right], \\
& c_{4}=\frac{1}{\pi^{4}}[-2148668+395264 \sqrt{2}+1790424 \sqrt{3}-(55319+209408 \sqrt{2}+74844 \sqrt{3}) \pi],
\end{aligned}
$$




$$
\begin{aligned}
& c_{5}=\frac{1}{\pi^{5}}[7497936-1769472 \sqrt{2}-5860188 \sqrt{3}+(178146+709632 \sqrt{2}+265032 \sqrt{3}) \pi], \\
& c_{6}=\frac{1}{\pi^{6}}[-15206904+4276224 \sqrt{2}+11209104 \sqrt{3}-(337140+1400832 \sqrt{2}+546264 \sqrt{3}) \pi], \\
& c_{7}=\frac{1}{\pi^{7}}[16604352-5308416 \sqrt{2}-11617344 \sqrt{3}+(347328+1492992 \sqrt{2}+606528 \sqrt{3}) \pi], \\
& c_{8}=\frac{1}{\pi^{8}}[-7537536+2654208 \sqrt{2}+5038848 \sqrt{3}-(150336+663552 \sqrt{2}+279936 \sqrt{3}) \pi], \\
& d_{2}=\frac{1}{\pi^{2}}\left[\frac{2249}{2}+2048 \sqrt{2}-3402 \sqrt{3}+\left(162+256 \sqrt{2}+81 \frac{\sqrt{3}}{2}\right) \pi\right] \\
& d_{3}=\frac{1}{\pi^{3}}\left[-32013-45056 \sqrt{2}+80676 \sqrt{3}-\left(3564+6144 \sqrt{2}+2025 \frac{\sqrt{3}}{2}\right) \pi\right] \text {, } \\
& d_{4}=\frac{1}{\pi^{4}}[342202+395264 \sqrt{2}-763506 \sqrt{3}+(31914+59648 \sqrt{2}+10287 \sqrt{3}) \pi], \\
& d_{5}=\frac{1}{\pi^{5}}[-1844604-1769472 \sqrt{2}+3718872 \sqrt{3}-(149040+299520 \sqrt{2}+54270 \sqrt{3}) \pi], \\
& d_{6}=\frac{1}{\pi^{6}}[5355216+4276224 \sqrt{2}-9873576 \sqrt{3}+(382968+820224 \sqrt{2}+156492 \sqrt{3}) \pi], \\
& d_{7}=\frac{1}{\pi^{7}}[-7967808-5308416 \sqrt{2}+13576896 \sqrt{3}-(513216+1161216 \sqrt{2}+233280 \sqrt{3}) \pi], \\
& d_{8}=\frac{1}{\pi^{8}}[4748544+2654208 \sqrt{2}-7558272 \sqrt{3}+(279936+663552 \sqrt{2}+139968 \sqrt{3}) \pi] .
\end{aligned}
$$

Proof. Let $\quad e_{c l}(x)=\cos (x)-1-d_{2} x^{2}-d_{3} x^{3}-d_{4} x^{4}-$ $d_{5} x^{5}-d_{6} x^{6}-d_{7} x^{7}-d_{8} x^{8}$ and $e_{c u}(x)=\cos (x)-1-c_{1} x-$ $c_{2} x^{2}-c_{3} x^{3}-c_{4} x^{4}-c_{5} x^{5}-c_{6} x^{6}-c_{7} x^{7}-c_{8} x^{8}$; then, $e_{c l}^{(9)}$ $(x)=e_{c u}^{(9)}(x)=\cos ^{(9)}(x)$.
It is obvious that $\cos ^{(9)}(x)=-\sin (x) \leq 0$ for $x \in(0,(\pi / 2))$.

By the definition of $e_{c l}(x)$ and $e_{c u}(x)$, we have

$$
\begin{aligned}
& e_{c u}(0)=e_{c u}\left(\frac{\pi}{6}\right)=e_{c u}\left(\frac{\pi}{4}\right)=e_{c u}\left(\frac{\pi}{3}\right)=e_{c u}\left(\frac{\pi}{2}\right)=e_{c u}^{\prime}\left(\frac{\pi}{6}\right)=e_{c u}^{\prime}\left(\frac{\pi}{4}\right)=e_{c u}^{\prime}\left(\frac{\pi}{3}\right)=e_{c u}^{\prime}\left(\frac{\pi}{2}\right)=0, \\
& e_{c l}(0)=e_{c l}\left(\frac{\pi}{6}\right)=e_{c l}\left(\frac{\pi}{4}\right)=e_{c l}\left(\frac{\pi}{3}\right)=e_{c l}\left(\frac{\pi}{2}\right)=e_{c l}^{\prime}(0)=e_{c l}^{\prime}\left(\frac{\pi}{6}\right)=e_{c l}^{\prime}\left(\frac{\pi}{4}\right)=e_{c l}^{\prime}\left(\frac{\pi}{3}\right)=0 .
\end{aligned}
$$

By Theorem 1, there exist $\eta_{j}, j=1,2$, such that

$$
\begin{gathered}
e_{c u}(x)=\frac{\cos ^{(9)}\left(\eta_{1}\right)}{9 !} x\left(x-\frac{\pi}{6}\right)^{2}\left(x-\frac{\pi}{4}\right)^{2}\left(x-\frac{\pi}{3}\right)^{2}\left(x-\frac{\pi}{2}\right)^{2} \leq 0, \\
e_{c l}(x)=\frac{\cos ^{(9)}\left(\eta_{2}\right)}{9 !} x^{2}\left(x-\frac{\pi}{6}\right)^{2}\left(x-\frac{\pi}{4}\right)^{2}\left(x-\frac{\pi}{3}\right)^{2}\left(x-\frac{\pi}{2}\right) \geq 0,
\end{gathered}
$$

which mean the conclusions are valid.

The proof of Theorem 3 is completed.

We propose the following refinements and improvements of inequalities (1)-(5).

Theorem 4. For $x \in(0,(\pi / 2))$, we have that 


$$
\begin{aligned}
& s_{l}(x)-\left(c_{u}(x)\right)^{(1 / 3)} \leq \frac{\sin (x)}{x}-(\cos (x))^{(1 / 3)} \leq s_{u}(x)-\left(c_{l}(x)\right)^{(1 / 3)}, \\
& \left(s_{l}(x)\right)^{3}-c_{u}(x) \leq\left(\frac{\sin (x)}{x}\right)^{3}-\cos (x) \leq\left(s_{u}(x)\right)^{3}-c_{l}(x), \\
& \frac{3 s_{l}(x)-c_{u}(x)-2}{3} \leq \frac{\sin (x)}{x}-\frac{2+\cos (x)}{3} \leq \frac{3 s_{u}(x)-c_{l}(x)-2}{3}, \\
& 2 s_{l}(x)+\frac{s_{l}(x)}{c_{u}(x)} \leq 2 \frac{\sin (x)}{x}+\frac{\tan (x)}{x} \leq 2 s_{u}(x)+\frac{s_{u}(x)}{c_{l}(x)} \\
& \left(s_{l}(x)\right)^{2}+\frac{s_{l}(x)}{c_{u}(x)} \leq\left(\frac{\sin (x)}{x}\right)^{2}+\frac{\tan (x)}{x} \leq\left(s_{u}(x)\right)^{2}+\frac{s_{u}(x)}{c_{l}(x)}, \\
& \frac{1}{\left(s_{u}(x)\right)^{2}}+\frac{c_{l}(x)}{s_{u}(x)} \leq\left(\frac{x}{\sin (x)}\right)^{2}+\frac{x}{\tan (x)} \leq \frac{1}{\left(s_{l}(x)\right)^{2}}+\frac{c_{u}(x)}{s_{l}(x)}, \\
& \frac{3}{s_{u}(x)}+c_{l}(x) \leq 3 \frac{x}{\sin (x)}+\cos (x) \leq \frac{3}{s_{l}(x)}+c_{u}(x),
\end{aligned}
$$

where $s_{l}(x), s_{u}(x), c_{l}(x)$, and $c_{u}(x)$ are defined in Theorems 2 and 3.

Proof. First, we prove inequality (44). By Theorems 2 and 3, we have

$$
\begin{aligned}
& s_{l}(x) \leq \frac{\sin (x)}{x} \leq s_{u}(x), \\
& c_{l}(x) \leq \cos (x) \leq c_{u}(x) .
\end{aligned}
$$

Therefore, we have $\left(c_{l}(x)\right)^{(1 / 3)} \leq(\cos (x))^{(1 / 3)} \leq$ $\left(c_{u}(x)\right)^{(1 / 3)} ; \quad$ then, $\quad s_{l}(x)-\left(c_{u}(x)\right)^{(1 / 3)} \leq(\sin (x) / x)-$ $(\cos (x))^{(1 / 3)} \leq s_{u}(x)-\left(c_{l}(x)\right)^{(1 / 3)}$. Inequality (44) is proved.

Second, we give the proof of inequality (48). For the same reason, we have $\left(s_{l}(x)\right)^{3}<(\sin (x) / x)^{3}<\left(s_{u}(x)\right)^{3}$ and $-c_{u}(x) \leq-\cos (x) \leq-c_{l}(x)$; then, $\quad\left(s_{l}(x)\right)^{3}-c_{u}(x) \leq$ $(\sin (x) / x)^{3}-\cos (x) \leq\left(s_{u}(x)\right)^{3}-c_{l}(x)$. That is to say, inequality (48) holds.

Third, we prove inequality (49).

It is easy to see that $\left(2+c_{l}(x) / 3\right) \leq$ $(2+\cos (x) / 3) \leq\left(2+c_{u}(x) / 3\right)$; then, inequality (49) is valid.

Fourth, we prove inequality (50). By Theorem 2, we have $c_{l}(x)=1+d_{2} x^{2}+d_{3} x^{3}+d_{4} x^{4}+d_{5} x^{5}+d_{6} x^{6}+d_{7} x^{7}+d_{8} x^{8}$, where $d_{i}, i=2,3, \ldots, 8$, are defined in Theorem 2. Then, $c_{l}(x)$ can be rewritten as

$$
\begin{aligned}
c_{l}(x)= & \left(x-x_{1}\right)\left(x-x_{2}\right)\left(x-x_{3}\right)\left(x-x_{4}\right)\left(x-x_{5}\right) \\
& \cdot\left(x-x_{6}\right)\left(x-x_{7}\right)\left(x-x_{8}\right),
\end{aligned}
$$

where $\quad x_{1} \approx-7.01106143, x_{2} \approx 5.18438798+2.25095656 i$, $x_{3} \approx 5.18438798-2.25095656 i, \quad x_{4} \approx 4.42109817, x_{5} \approx-$ $4.444313683+1.43538013 i, x_{6} \approx-4.444313683-1.43538013 i$, $x_{7} \approx 1.57079633, x_{8} \approx-1.56923028$.
Then, $x \in(0,(\pi / 2))$, and we have $c_{l}(x)>0$. For the same reason, we can prove $s_{l}(x)>0, x \in(0,(\pi / 2))$. It is obvious that $c_{u}(x)>0$ and $s_{u}(x)>0$.

By inequality (55) and the results of $c_{l}(x)>0$, $s_{l}(x)>0, c_{u}(x)>0$, and $s_{u}(x)>0$, we have

$$
\begin{gathered}
2 s_{l}(x) \leq 2 \frac{\sin (x)}{x} \leq 2 s_{u}(x), \\
\frac{s_{l}(x)}{c_{u}(x)} \leq \frac{\tan (x)}{x} \leq \frac{s_{u}(x)}{c_{l}(x)} .
\end{gathered}
$$

Hence, inequality (50) holds.

Fifth, we prove inequality (51). By the result of $s_{l}(x)>0$ and $s_{u}(x)>0$, we have $\left(s_{l}(x)\right)^{2}<(\sin (x) / x)^{2}<\left(s_{u}(x)\right)^{2}$. And because of the above results of $\left(s_{l}(x) / c_{u}\right.$ $(x)) \leq(\tan (x) / x) \leq\left(s_{u}(x) / c_{l}(x)\right)$, we have that inequality (51) holds.

Sixth, we prove inequality (52). By the result of $s_{l}(x)>0$ and $s_{u}(x)>0$, we have $\left(s_{l}(x)\right)^{2}<(\sin (x) / x)^{2}<\left(s_{u}(x)\right)^{2}$. And because of the results of $\left(s_{l}(x) / c_{u}(x)\right) \leq$ $(\tan (x) / x) \leq\left(s_{u}(x) / c_{l}(x)\right)$ and $s_{l}(x)>0, s_{u}(x)>0, c_{l}(x)>$ $0, c_{u}(x)>0$, we have $\left(c_{l}(x) / s_{u}(x)\right)<(x / \tan (x))<$ $\left(c_{u}(x) / s_{l}(x)\right)$. Therefore, inequality (52) is proved.

At last, we prove inequality (53). By the results of $s_{l}(x)>0, s_{u}(x)>0$ and inequality (54), we have $1 / s_{u}(x)<x / \sin (x)<1 / s_{l}(x)$. And because of inequality (55), inequality (53) holds.

The proof of Theorem 4 is completed.

Theorems 2 and 3 give the new bounds of $\sin c(x)$ and $\cos (x)$ based on the interpolation and approximation method. Theorem 4 gives the improvements and refinements of Mitrinović-Cusa's and related inequalities using the new bounds of $\sin c(x)$ and $\cos (x)$. Next, we try to obtain the new improvements of Mitrinović-Cusa's 
inequalities directly based on the interpolation and approximation method.

Theorem 5. For $x \in(0,(\pi / 2))$, we have

$$
g_{2} x^{2}+g_{3} x^{3}+g_{4} x^{4}+g_{5} x^{5}+g_{6} x^{6} \leq \frac{\sin (x)}{x}-\frac{2+\cos (x)}{3} \leq f_{1} x+f_{2} x^{2}+f_{3} x^{3}+f_{4} x^{4}+f_{5} x^{5}+f_{6} x^{6}
$$

where

$$
\begin{aligned}
& f_{1}=\frac{1}{\pi^{2}}\left[208-2560 \sqrt{2}+5103 \frac{\sqrt{3}}{2}-\left(\frac{753}{2}-128 \sqrt{2}\right) \pi-\left(\frac{4}{3}+32 \frac{\sqrt{2}}{3}+27 \frac{\sqrt{3}}{2}\right) \pi^{2}\right], \\
& f_{2}=\frac{1}{\pi^{3}}\left[-3296+37888 \sqrt{2}-37179 \sqrt{3}+\left(\frac{16304}{3}-5888 \frac{\sqrt{2}}{3}\right) \pi+\left(64 / 3+448 \frac{\sqrt{2}}{3}+405 \frac{\sqrt{3}}{2}\right) \pi^{2}\right], \\
& f_{3}=\frac{1}{\pi^{4}}\left[20560-217600 \sqrt{2}+211410 \sqrt{3}-\left(30962-34688 \frac{\sqrt{2}}{3}\right) \pi-\left(\frac{404}{3}+2464 \frac{\sqrt{2}}{3}+1188 \sqrt{3}\right) \pi^{2}\right], \\
& f_{4}=\frac{1}{\pi^{5}}\left[-62976+608256 \sqrt{2}-586116 \sqrt{3}+\left(\frac{258824}{3}-98816 \sqrt{2} / 3\right) \pi+\left(\frac{1256}{3}+6656 \frac{\sqrt{2}}{3}+3402 \sqrt{3}\right) \pi^{2}\right], \\
& f_{5}=\frac{1}{\pi^{6}}\left[94464-829440 \sqrt{2}+793152 \sqrt{3}-(117472-45568 \sqrt{2}) \pi-(640+2944 \sqrt{2}+4752 \sqrt{3}) \pi^{2}\right], \\
& f_{6}=\frac{1}{\pi^{7}}\left[-55296+442368 \sqrt{2}-419904 \sqrt{3}+(62592-24576 \sqrt{2}) \pi+(384+1536 \sqrt{2}+2592 \sqrt{3}) \pi^{2}\right], \\
& g_{2}=\frac{1}{\pi^{3}}\left[32-3072 \sqrt{2}+3645 \sqrt{3}-\left(\frac{1768}{3}-256 \frac{\sqrt{2}}{3}\right) \pi-\left(64 \frac{\sqrt{2}}{3}+27 \frac{\sqrt{3}}{2}\right) \pi^{2}\right], \\
& g_{3}=\frac{1}{\pi^{4}}\left[-448+40960 \sqrt{2}-92583 \sqrt{3} / 2+\left(\frac{14129}{2}-4096 \frac{\sqrt{2}}{3}\right) \pi+\left(256 \sqrt{2}+351 \frac{\sqrt{3}}{2}\right) \pi^{2}\right], \\
& g_{4}=\frac{1}{\pi^{5}}\left[2336-195584 \sqrt{2}+215055 \sqrt{3}-\left(\frac{95839}{3}-21760 \frac{\sqrt{2}}{3}\right) \pi-\left(3392 \frac{\sqrt{2}}{3}+837 \sqrt{3}\right) \pi^{2}\right], \\
& g_{5}=\frac{1}{\pi^{6}}\left[-5376+399360 \sqrt{2}-431568 \sqrt{3}+(63248-15872 \sqrt{2}) \pi+(2176 \sqrt{2}+1728 \sqrt{3}) \pi^{2}\right], \\
& g_{6}=\frac{1}{\pi^{7}}\left[4608-294912 \sqrt{2}+314928 \sqrt{3}-(45840-12288 \sqrt{2}) \pi-(1536 \sqrt{2}+1296 \sqrt{3}) \pi^{2}\right] .
\end{aligned}
$$

Proof. Let $f_{s c}(x)=(\sin (x) / x)-(2+\cos (x) / 3), \quad e_{l}(x)=$ $f_{s c}(x)-g_{2} x^{2}-g_{3} x^{3}-g_{4} x^{4}-g_{5} x^{5}-g_{6} x^{6}$, and $e_{u}(x)=$ $f_{s c}(x)-f_{1} x-f_{2} x^{2}-f_{3} x^{3}-f_{4} x^{4}-f_{5} x^{5}-f_{6} x^{6}$; then,

$$
\begin{aligned}
e_{l}^{(7)}(x) & =e_{u}^{(7)}(x)=\sin c^{(7)}(x)-\frac{1}{3} \sin (x) \\
& =\frac{\left(7 x^{6}-210 x^{4}+2520 x^{2}-5040\right) \sin (x)+\left(-x^{7}+42 x^{5}-840 x^{3}+5040 x\right) \cos (x)}{x^{8}}-\frac{1}{3} \sin (x) .
\end{aligned}
$$


TABLE 1: Maximum errors between $(\sin (x) / x)^{3}-\cos (x)$ and its bounds (Mitrinovićs inequality).

\begin{tabular}{lcr}
\hline Method & & Error \\
& MaxError $_{\text {low }}$ & MaxError $_{\text {upp }}$ \\
\hline Mortici [42] (inequality (24)) & $3.4808 \times 10^{-2}$ & $1.4744 \times 10^{-1}$ \\
Maleševic et al. [43] (inequality (30), $n=2)$ & $5.6770 \times 10^{-4}$ & $5.2314 \times 10^{-3}$ \\
The results of this paper (inequality (48)) & $3.2409 \times 10^{-8}$ & $3.1707 \times 10^{-8}$ \\
\hline
\end{tabular}

TABLE 2: Maximum errors between $(\sin (x) / x)-(2+\cos (x) / 3)$ and its bounds (Cusa's inequality).

\begin{tabular}{lcr}
\hline Method & & Error \\
\hline Bhayo and sandor [33] (inequality (6)) & MaxError $_{\text {low }}$ & MaxError $_{\text {upp }}$ \\
Mortici [42] (inequality (25)) & $1.1614 \times 10^{-2}$ & $9.1985 \times 10^{-3}$ \\
Maleševic et al. [43] (inequality (31), $n=2)$ & $3.7644 \times 10^{-3}$ & $1.9749 \times 10^{-4}$ \\
The results of this paper (inequality (49)) & $5.9603 \times 10^{-6}$ & $1.1839 \times 10^{-7}$ \\
The results of this paper (inequality (56)) & $1.0803 \times 10^{-8}$ & $1.0701 \times 10^{-8}$ \\
\hline
\end{tabular}

TABle 3: Maximum errors between $2(\sin (x) / x)+(\tan (x) / x)$ and its bounds (Huygens' inequality).

\begin{tabular}{lcr}
\hline Method & & Error \\
& MaxError $_{\text {low }}$ & MaxError $_{\text {upp }}$ \\
\hline Huygens [3] (inequality (2)) & $7.9812 \times 10^{2}$ & - \\
Chen and Cheung [37] (inequality (11)) & $6.9173 \times 10^{1}$ & $1.0539 \times 10^{-1}$ \\
Chen and Cheung [37] (inequality (12)) & $1.5550 \times 10^{2}$ & $2.0761 \times 10^{-1}$ \\
Chen and Paris [39] (inequality (19)) & $6.1807 \times 10^{0}$ & $8.3747 \times 10^{-3}$ \\
Mortici [42] (inequality (26)) & $2.9643 \times 10^{-3}$ & $3.4633 \times 10^{2}$ \\
Maleševic et al. [43] (inequality (32), $n=2)$ & $1.3520 \times 10^{0}$ & $4.4188 \times 10^{0}$ \\
The results of this paper (inequality (50)) & $3.8724 \times 10^{-7}$ & $7.1581 \times 10^{-4}$ \\
\hline
\end{tabular}

TABLe 4: Maximum errors between $(\sin (x) / x)^{2}+(\tan (x) / x)$ and its bounds (Wilker's inequality).

\begin{tabular}{lcc}
\hline Method & & Error \\
& MaxError $_{\text {low }}$ & MaxError $_{\text {upp }}$ \\
\hline Wilker and Porblem [4] (inequality (3)) & $7.9826 \times 10^{2}$ & - \\
Sumner et al. [36] (inequality (10)) & $2.6424 \times 10^{-2}$ & $6.5686 \times 10^{1}$ \\
Chen and Cheung [37] (inequality (13)) & $1.8874 \times 10^{2}$ & $2.4457 \times 10^{-1}$ \\
Chen and Cheung [37] (inequality (14)) & $1.4653 \times 10^{2}$ & $1.9695 \times 10^{-1}$ \\
Nenezic et al. [38] (inequality (17)) & $3.5720 \times 10^{1}$ & $3.9383 \times 10^{-3}$ \\
Nenezic et al. [38] (inequality (18)) & $5.7271 \times 10^{-3}$ & $3.0139 \times 10^{-3}$ \\
Chen and Paris [39] (inequality (20)) & $2.3923 \times 10^{0}$ & $3.9383 \times 10^{-3}$ \\
Mortici [42] (inequality (27)) & $8.7474 \times 10^{2}$ & $5.5813 \times 10^{2}$ \\
Maleševic et al. [43] (inequality (33), $n=2)$ & $3.5293 \times 10^{0}$ & $2.8103 \times 10^{1}$ \\
The results of this paper (inequality (51)) & $3.8636 \times 10^{-7}$ & $7.1581 \times 10^{-4}$ \\
\hline
\end{tabular}

We let $h(x)=\left(7 x^{6}-210 x^{4}+2520 x^{2}-5040\right) \sin (x)+$ $\left(-x^{7}+42 x^{5}-840 x^{3}+5040 x\right) \cos (x)$; then, we have $h^{\prime}(x)=-5 x^{7}-x^{8} \cos (x)<0, x \in(0,(\pi / 2))$. Then, $h(x)$ is a monotone decreasing function in $(0,(\pi / 2))$, and we have $h(x) \leq h(0)=0$; then, $\sin c^{(7)}(x) \leq 0$ for $x \in(0,(\pi / 2))$. Therefore, we have $e_{l}^{(7)}(x)=e_{u}^{(7)}(x)=f_{s c}^{(7)}(x)=$ $\sin c^{(7)}(x)-(1 / 3) \sin (x)<0$.

By the definition of $e_{l}(x)$ and $e_{u}(x)$, we have

$$
\begin{aligned}
& e_{l}(0)=e_{l}\left(\frac{\pi}{4}\right)=e_{l}\left(\frac{\pi}{3}\right)=e_{l}\left(\frac{\pi}{2}\right)=e_{l}^{\prime}(0)=e_{l}^{\prime}\left(\frac{\pi}{4}\right)=e_{l}^{\prime}\left(\frac{\pi}{3}\right)=0, \\
& e_{u}(0)=e_{u}\left(\frac{\pi}{4}\right)=e_{u}\left(\frac{\pi}{3}\right)=e_{u}\left(\frac{\pi}{2}\right)=e_{u}^{\prime}\left(\frac{\pi}{4}\right)=e_{u}^{\prime}\left(\frac{\pi}{3}\right)=e_{u}^{\prime}\left(\frac{\pi}{2}\right)=0 .
\end{aligned}
$$

By Theorem 1 , there exist $\theta_{j}, j=1,2$, such that 
TABle 5: Maximum errors between $(x / \sin (x))^{2}+(x / \tan (x))$ and its bounds (Wu and Srivastava's inequality).

\begin{tabular}{lcr}
\hline Method & & Error \\
\hline Wu and Srivastava [5] (inequality (4)) & MaxError $_{\text {low }}$ & MaxError $_{\text {upp }}$ \\
Chen and Cheung [37] (inequality (15)) & $4.6615 \times 10^{-1}$ & - \\
Chen and Cheung [37] (inequality (16)) & - & $2.1552 \times 10^{2}$ \\
Chen and Paris [39] (inequality (21)) & - & $1.0111 \times 10^{2}$ \\
Wang [40] (inequality (22)) & $1.0560 \times 10^{-1}$ & $6.4478 \times 10^{1}$ \\
Mortici [42] (inequality (28)) & $2.9416 \times 10^{-1}$ & $9.4934 \times 10^{-2}$ \\
Maleševic et al. [43] (inequality (34), $m=3$ ) & $1.9612 \times 10^{-1}$ & - \\
The results of this paper (inequality (52)) & $6.9338 \times 10^{-2}$ & $9.6995 \times 10^{-3}$ \\
\hline
\end{tabular}

TABle 6: Maximum errors between $3(x / \sin (x))+(x / \tan (x))$ and its bounds (Neuman and Sandor's inequality).

\begin{tabular}{lcr}
\hline Method & & Error \\
& MaxError $_{\text {low }}$ & MaxError $_{\text {upp }}$ \\
\hline Neuman and Sandor [6] (inequality (5)) & $7.1080 \times 10^{-1}$ & - \\
Jiang et al. [41] (inequality (23)) & $3.2381 \times 10^{-1}$ & $7.4286 \times 10^{-2}$ \\
Mortici [42] (inequality (29)) & $3.1910 \times 10^{-2}$ & - \\
Maleševic et al. [43] (inequality (35), $m=3$ ) & $63.1910 \times 10^{-2}$ & $4.1471 \times 10^{-3}$ \\
The results of this paper (inequality (53)) & $3.2982 \times 10^{-8}$ & $3.2411 \times 10^{-8}$ \\
\hline
\end{tabular}

$$
\begin{aligned}
& e_{l}(x)=\frac{f_{s c}^{(7)}\left(\theta_{1}\right)}{7 !} x^{2}\left(x-\frac{\pi}{4}\right)^{2}\left(x-\frac{\pi}{3}\right)^{2}\left(x-\frac{\pi}{2}\right) \geq 0, \\
& e_{u}(x)=\frac{f_{s c}^{(7)}\left(\theta_{2}\right)}{7 !} x\left(x-\frac{\pi}{4}\right)^{2}\left(x-\frac{\pi}{3}\right)^{2}\left(x-\frac{\pi}{2}\right)^{2} \leq 0,
\end{aligned}
$$

which mean the conclusions are valid.

\section{Conclusions and Analysis}

In this paper, we give the new results of Mitrinović-Cusa's and related inequalities, including Wilker's, Huygens', Wu-Srivastava's, and Neuman-Sandor's inequalities. Theorems 2 and 3 present the novel polynomial bounds of $\sin c(x)$ and $\cos (x)$ functions using the interpolation and approximation method. Theorem 4 gives the new refinements and improvements of the above five inequalities based on the results of $\sin c(x)$ and $\cos (x)$. In order to compare our results with the previous methods, we introduce the concept of the maximum error. The maximum error is the most important index to measure the upper and lower bounds of an inequality (a function). MaxError ${ }_{\text {low }}$ denotes the maximum error between a function and its lower bound. Similarly, MaxError ${ }_{\text {low }}$ denotes the maximum error between a function and its upper bound. Tables 1-6 give the maximum errors of the upper and lower bounds of the above five inequalities. We consider both sides of Mitrinović-Cusa's inequality separately, which is why there are six tables for five inequalities. It is obvious that the results of this paper are superior to the previous conclusions.

For Mitrinović's inequality, many researchers focused on the equivalent form of this inequality, that is, the inequality $(\sin (x) / x)^{3}-\cos (x)>0$. Table 1 gives the comparison of the maximum errors between $(\sin (x) / x)^{3}-\cos (x)>0$ and its bounds for different methods. In inequality (40), the maximum error between the function $(\sin (x) / x)^{3}-$ $\cos (x)>0$ and the lower bound is only $3.2409 \times 10^{-8}$, and the maximum error of the upper bound is $3.1707 \times 10^{-8}$. It is easy to see that the maximum errors of this paper are the smallest of all methods. For Cusa's inequality, Wilker's inequality, Huygens' inequality, $\mathrm{Wu}$-Srivastava's inequality, and Neuman-Sandor's inequality, the same conclusions can be obtained from Tables 2 to 6 .

In this paper, we not only consider the equivalent form of Mitrinovićs inequality but also consider the lower and upper bounds of the function $(\sin (x) / x)-\cos (x)^{(1 / 3)} \mathrm{di}-$ rectly. Inequality (44) gives the new improvement of Mitrinovićs inequality. The maximum error between the function $(\sin (x) / x)-\cos (x)^{(1 / 3)}$ and the lower bound is only $1.0834 \times 10^{-8}$, and the maximum error of the upper bound is $6.8129 \times 10^{-8}$.

Tables 1-6 show that the results of this paper are far superior to the previous conclusions. Among the previous conclusions, only the conclusions of Maleševic et al. [43] are close to the results of this paper for Cusa's inequality in Table 2. In this paper, the maximum error between $(\sin (x) / x)-(2+\cos (x) / 3)$ and its lower bound is $1.0803 \times 10^{-8}$, and the maximum error between $(\sin (x) / x)-(2+\cos (x) / 3)$ and its upper bound is $1.0701 \times 10^{-8}$. The maximum error of the lower bound is $5.9603 \times 10^{-6}$, and the maximum error of the upper bound is $1.1839 \times 10^{-7}$ in Maleševiç et al. [43]. Although the maximum errors of Maleševic et al. [43] are close to our results, we can find that the degree of the bounds is 8 in inequality (49), and the degree of the upper bound reaches 10 in inequality (33) (when $n=2$ ).

Another advantage of our results is that the form of the bounds is relatively simple. The bounds are all polynomial 
functions, and there are no other functions. In previous conclusions, many bounds contain not only polynomial functions but also trigonometric functions, for example, $\sin (x), \cos (x)$, and $\tan (x)$. In general, the polynomial function as the upper and lower bounds of the trigonometric function is more effective than the trigonometric function as the upper and lower bounds of the trigonometric function.

At the end of this paper, we try to obtain the bounds of $(\sin (x) / x)-(2+\cos (x) / 3)$ directly using the interpolation and approximation theorem. Theorem 5 gives the new upper and lower bounds of the function $(\sin (x) / x)-(2+$ $\cos (x) / 3)$. The last row of Table 2 gives the maximum errors of inequality (56). The maximum error between the function $(\sin (x) / x)-(2+\cos (x) / 3)$ and the lower bound is $5.8244 \times 10^{-7}$, and the maximum error of the upper bound is $3.2174 \times 10^{-6}$. Table 2 shows that the maximum errors are close to the results of inequality (49). Although the maximum errors of are bigger than the maximum errors of inequality (49), the degree of the bounds is 6 in inequality (56), and the degree of the bounds is 8 in inequality (49). Similarly, we can obtain the upper and lower bounds of other functions directly using the interpolation and approximation theorem.

\section{Data Availability}

No data were used to support this study.

\section{Conflicts of Interest}

The authors declare that they have no conflicts of interest.

\section{Acknowledgments}

This work was partially supported by the National Natural Science Foundation of China (no. 11701152), Key Research Project of Colleges and Universities in Henan Province (no. 20A520016), and the Ph.D. Foundation of Henan Polytechnic University (no. B2017-44).

\section{References}

[1] D. S. Mitrinovi, "Limitations en module d'une fonction homographique sur un cercle," Univerzitet U Beogradu. Publikacije Elektrotehnic kog Fakulteta. Serija Matematika I Fizika, vol. 143-155, pp. 3-4, 1965.

[2] D. S. Mitrinovi, P. M. Vasi, Analytic Inequalities, SpringerVerlag, Berlin, Germany, 1970.

[3] C. Huygens, Oeuvres Completes 1888-1940, Socite Hollondaise des Science, Haga, Gothenburg, 1940.

[4] J. B. Wilker and e Porblem, The American Mathematical Monthly, vol. 96, no. 55, p. 3306, 1989.

[5] S. H. Wu and H. M. Srivastava, "A weighted and exponential generalization of wilker's inequality and its applications," Integral Transforms and Special Functions, vol. 18, no. 8, pp. 525-535, 2007.

[6] E. Neuman and J. Sándor, "On some inequalities involving trigonometric and hyperbolic functions with emphasis on the cusa-huygens, wilker, and huygens inequalities," Mathematical Inequalities \& Applications, vol. 13, no. 4, pp. 715-723, 2010.
[7] E. Neuman and Wilker, "Wilker and Huygens- type inequalities for the generalized trigonometric and for the generalized hyperbolic functions," Applied Mathematics and Computation, vol. 230, pp. 211-217, 2014.

[8] L. Zhu, "On wilker-type inequalities," Mathematical Inequalities \& Applications, vol. 10, no. 4, pp. 727-731, 2007.

[9] L. Zhu, "Some new inequalities of the huygens type," Computers \& Mathematics with Applications, vol. 58, no. 6, pp. 1180-1182, 2009.

[10] L. Zhu, "A source of inequalities for circular functions," Computers and Mathematics with Applications, vol. 58, no. 10, pp. 1998-2004, 2009.

[11] L. Zhu, "An unity of mitrinovic-adamovic and cusa-huygens inequalities and the analogue for hyperbolic functions," Revista de la Real Academia de Ciencias Exactas, Físicas y Naturales. Serie A. Matemáticas, vol. 113, no. 4, pp. 33993412, 2019.

[12] L. Zhu, "New cusa-huygens type inequalities," AIMS Mathematics, vol. 5, no. 5, pp. 5320-5331, 2020.

[13] L. Zhu, "New inequalities of Wilker's type for circular functions," AIMS Mathematics, vol. 5, no. 5, pp. 4874-4888, 2020.

[14] L. Zhu, Z. J. Sun, and Z. Sun, "Refinements of huygens- and wilker- type inequalities," AIMS Mathematics, vol. 5, no. 4, pp. 2967-2978, 2020.

[15] L. N. Zhang and X. S. Ma, "New refinements and improvements of some trigonometric inequalities based on pade approximant," Journal of Mathematics, vol. 2020, Article ID 2753691, 2020.

[16] S. H. Wu and A. Baricz, "Generalizations of mitrinović, adamović and lazarević's inequalities and their applications," Publicationes Mathematicae, vol. 75, no. 3-4, pp. 447-458, 2009.

[17] S.-H. Wu, S.-G. Li, and M. Bencze, "Sharpened versions of Mitrinović-Adamović, lazarević and wilkers inequalities for trigonometric and hyperbolic functions," Journal of Nonlinear Sciences and Applications, vol. 09, no. 05, pp. 2688-2696, 2016.

[18] J. S, ndor and M. Bencze, "On huygen's trigonometric inequality," RGMIA Resident Representative Collection, vol. 8, no. 3, 2005.

[19] J. S, ndor, "On huygens' inequalities and the theory of means," International Journal of Mathematics and Mathematical Sciences, vol. 2012, Article ID 597490, 9 pages, 2012.

[20] J. S, ndor and R. Ol, h-G, l, "On cusa-huygens type trigonometric and hyperbolic inequalities," Acta Universitatis Sapientiae Mathematica, vol. 4, no. 2, 2012.

[21] J. S, ndor, "Sharp cusa-huygens and related inequalities," Notes on Number Theory and Discrete Mathematics, vol. 19, no. 1, pp. 50-54, 2013.

[22] C. Mortici, "A subtly analysis of wilker inequality," Applied Mathematics and Computation, vol. 231, pp. 516-520, 2014.

[23] Z. H. Yang and Y. M. Chu, "A note on jordan, adamovicmitrinovic, and cusa inequalities," Abstract and Applied Analysis, vol. 2014, Article ID 364076, 12 pages, 2014.

[24] Z.-H. Yang, Y.-M. Chu, and X.-H. Zhang, "Sharp cusa type inequalities with two parameters and their applications," Applied Mathematics and Computation, vol. 268, pp. 11771198, 2015.

[25] C. P. Chen and J. Sandor, "Inequality chains for wilker, huygens and lazarevic type inequalities," Journal of Mathematical Inequalities, vol. 8, no. 1, 2014.

[26] X. D. Chen, J. P. Ma, J. Y in, and Y. G. Wang, "A two-point-padèapproximant-based method for bounding some trigonometric 
functions," Journal of Inequalities and Applications, vol. 1, p. 140, 2018.

[27] X. D. Chen, J. Y. Ma, and Y. X. Li, "Approximating trigonometric functions by using exponential inequalities," Journal of Inequalities and Applications, vol. 2019, no. 1, p. 59, 2019.

[28] L. G. Huang, L. Yin, Y. L. Wang, and X. L. Lin, "Some wilker and cusa type inequalities for generalized trigonometric and hyperbolic functions," Journal of Inequalities and Applications, vol. 2018, no. 1, p. 52, 2018.

[29] Y. J. Bagul and C. Chesneau, "Refined forms of oppenheim and cusa-huygens type inequalities," Acta et Commentationes Universitatis Tartuensis de Mathematica, vol. 24, no. 2, pp. 183-194, 2020.

[30] C. Chesneau and M. Kosti, B. Male, evi, B. Banjac and Y. J. Bagul, "New refinements of cusa-huygens inequality," 2020, https://arxiv.org/abs/2009.01688v1.

[31] B. Male, evi, M. Makragi, "A method for proving some inequalities on mixed trigonometric polynomial functions," Journal of Mathematical Inequalities, vol. 10, no. 3, pp. 849-876, 2016.

[32] T. Lutovac and B. Male, evi, C. Mortici, "The natural algorithmic approach of mixed trigonometric-polynomial problems," Journal of Inequalities and Applications, vol. 116, no. 1-16, 2017.

[33] B. A. Bhayo and J. Sándor, “On Jordan's, Redheffer's and Wilker's inequality," Mathematical Inequalities \& Applications, vol. 19, no. 3, pp. 823-839, 2016.

[34] Z. H. Yang, "Refinements of mitrinović-cusa inequality," 2012, https://arxiv.org/abs/1206.4911.

[35] Z. H. Yang, "Unification and refinements of Jordan, adamović-mitrinović and and cusa's inequalities," 2013, https://arxiv.org/abs/1304.3180.

[36] J. S. Sumner, A. A. Jagers, M. Vowe, and J. Anglesio, "Inequalities involving trigonometric functions," The American Mathematical Monthly, vol. 98, no. 3, pp. 264-267, 1991.

[37] C. P. Chen and W. S. Cheung, "Sharpness of wilker and huygens type inequalities," Journal of Inequalities and Applications, vol. 2012, no. 1, p. 72, 2012.

[38] M. Nenezic, B. Malesevic, and C. Mortici, "New approximations of some expressions involving trigonometric functions," Applied Mathematics and Computation, vol. 283, pp. 299-315, 2016.

[39] C. P. Chen and R. Paris, "On the wilker and huygens-type inequalities," 2016, https://arxiv.org/pdf/1602.00436.

[40] Z. H. Wang, "A new wilker-type inequality," Journal of Yibin University, vol. 6, no. 6, pp. 21-22, 2007.

[41] W.-D. Jiang, Q.-M. Luo, and F. Qi, "Refinements and sharpening of some huygens and wilker type inequalities," Turkish Journal of Analysis and Number Theory, vol. 2, no. 4, pp. 134-139, 2014.

[42] C. Mortici, "The natural approach of wilker-cusa-huygens inequalities," Mathematical Inequalities \& Applications, vol. 14, no. 3, pp. 535-541, 2011.

[43] B. Male, evi, T. Lutovac and M. Ra, ajski and C. Mortici, "Extensions of the natural approach to refinements and generalizations of some trigonometric inequalities," Advances in Difference Equations, vol. 2018, no. 1, p. 90, 2018.

[44] X.-D. Chen, J. Shi, Y. Wang, and P. Xiang, "A new method for sharpening the bounds of several special functions," Results in Mathematics, vol. 72, no. 1-2, pp. 695-702, 2017.

[45] L. N. Zhang and X. S. Ma, "New refinements and improvements of Jordan's inequality," Mathematics, vol. 7, no. 284, 2018.
[46] L. N. Zhang and X. S. Ma, "New polynomial bounds for Jordan's and kober's inequalities based on the interpolation and approximation method," Mathematics, vol. 7, no. 746, 2019.

[47] P. J. Davis, Interpolation and Approximation, Dover Publications, New York, NY, USA, 1975. 